\title{
Simplified Biorelevant Media for Screening Dissolution Performance of Poorly Soluble Drugs
}

\author{
Thomas Zoeller and Sandra Klein ${ }^{1}$ \\ Institute of Pharmaceutical Technology, Johann Wolfgang Goethe University, 9 Max von Laue \\ Street, Frankfurt am Main 60438, Germany
}

\section{INTRODUCTION}

S imulation of gastrointestinal conditions is essential to adequately predict the in vivo behavior of poorly soluble drugs. Simulating small intestinal conditions with biorelevant media such as fasted state simulated intestinal fluid (FaSSIF) and fed state simulated intestinal fluid (FeSSIF) has become standard practice in many dissolution laboratories (1-6). However, due to their complex composition, these media are expensive and, to date, need to be prepared on the day of the experiment.

The aim of the present study was to develop media that are easier to prepare and are stable over a longer period, but can still serve the purpose of forecasting in vivo performance. Criteria for developing simplified test media also include cost-effectiveness and the ability to adequately reflect the physicochemical properties of the biorelevant media FaSSIF or FeSSIF (see Table 1). Another objective was to create mixed micelles like those formed by natural bile components. For this purpose, sodium taurocholate and lecithin were replaced with different types and concentrations of surfactants, and the physicochemical properties of the resulting mixtures were screened. Subsequently, simplified media with corresponding physicochemical properties were used for dissolution experiments. Since their oral bioavailability and dissolution rate performance have been reported to depend on the presence of dissolution enhancers $(1,3$, 7-10), ketoconazole, glyburide and tamoxifen citrate were selected as model drugs for these experiments. Based on these observations, it was assumed that drug release from their formulations would be sensitive to the composition of the test media. Thus, they appeared to be optimal candidates to prove the applicability of the new set of media.

\section{MATERIALS AND METHODS \\ Materials}

Brij ${ }^{\circledR} 35$ (polyoxyethyleneglycol dodecyl ether), lot \# 602D0048B (Uniqema, Wilton, UK); Span ${ }^{\circledR} 80$ (sorbitan monooleate), lot \# 813530 (Th. Goldschmidt, Essen, Germany); Tween ${ }^{\circledR} 20$ (polyoxyethylene sorbitan monolaurate), sample \# SURF17728 (ICl surfactants,

${ }^{1}$ Corresponding author.

Dissolution Technologies I NOVEMBER 2007
Middlesbrough, England); Tween ${ }^{\circledR} 40$ (polyoxyethylene sorbitan monopalmitate), sample \# SURF7168 (ICI surfactants, Middlesbrough, England); Tween ${ }^{\circledR} 60$ (polyoxyethylene sorbitan monostearate), lot \# S753320040 (Cognis GmbH, Duesseldorf, Germany); Tween ${ }^{\circledR} 80$ (polyoxyethylene sorbitan monooleate), lot \# 813530 (Th. Goldschmidt, Essen, Germany); and Texapon ${ }^{\circledR} \mathrm{K} 12 \mathrm{P}$ (sodium dodecyl sulfate), lot \# CS70910002 (Cognis GmbH, Duesseldorf, Germany) were all of analytical grade and donated by their manufacturers. Triton ${ }^{\circledR}$ X-100 (polyoxyethylene octyl phenyl ether) was purchased from Merck KGaA, Darmstadt, Germany, and triethanolamine ( $\mathrm{min} 98 \%$ ), lot \# 38H0128, was purchased from Sigma Aldrich, Steinheim, Germany.

Ketoconazole standard (lot \# 10004924) was a donation of Janssen Pharmaceutica (Beerse, Belgium). Glyburide (lot \# 024K0701) was purchased from Sigma Aldrich, Steinheim, Germany. Tamoxifen citrate (lot \# SRP 01106t) was obtained from Sequoia Research Products Ltd., Pangbourne, UK. Nizoral ${ }^{\circledR}$, lot \# 03EL8333 (Janssen-Cilag GmbH, Neuss, Germany); Euglucon ${ }^{\circledR}$ N, lot \# 40E500 (Aventis Pharma Deutschland GmbH, Frankfurt, Germany); and Tamoxifen Hexal 20 mg, lot \# 61MR19 (HEXAL AG, Holzkirchen, Germany) were purchased commercially. White opaque gelatin capsules (Wepa Nr. 35758 according to DAB 10; size $1 ; 0.5 \mathrm{~mL}$ ) were purchased from Wepa Apothekenbedarf GmbH \& Co KG, Hillscheid, Germany. Sodium taurocholate (PCA code 2012), lot \# 2003040161, was acquired from Prodotti Chimici e Alimentari S.P.A., Basaluzzo (AL), Italy, and egg-phosphatidylcholine, Lipoid EPCS (99.1\% pure), was kindly donated by Lipoid $\mathrm{GmbH}$, Ludwigshafen, Germany. All other compounds were of analytical grade and purchased commercially.

\section{Media Preparation}

The biorelevant media FaSSIF and FeSSIF (see Table 1 for compositions) were prepared on the day of the experiment. FaSSIF was prepared as follows: $3.3 \mathrm{~g}$ sodium taurocholate was dissolved in approximately $500 \mathrm{~mL}$ of the blank FaSSIF. The weight of this mixture was checked and noted ("weight 1"). Then $11.8 \mathrm{~mL}$ of a methylene chloride solution containing $100 \mathrm{mg} / \mathrm{mL}$ lecithin (= $1.18 \mathrm{~g}$ lecithin, "weight 2") was added. This produced an emulsion (i.e., the resulting product was turbid). The methylene chloride was then evaporated under vacuum using a 
Rotavap (type R-114, Buechi, Essen, Germany) at a temperature of about $40^{\circ} \mathrm{C}$. About $10 \mathrm{~min}$ at $500 \mathrm{mbar}$ followed by $30 \mathrm{~min}$ at about $50 \mathrm{mbar}$ led to complete removal of the methylene chloride. The result was a clear, micellar solution having no perceptible odor of methylene chloride. After cooling to room temperature, the weight of the solution was checked again. The water lost to evaporation was replaced with demineralized water to obtain a total weight corresponding to the sum of "weight 1" and "weight 2." Finally, the volume was brought to $2 \mathrm{~L}$ with blank FaSSIF.

Likewise, FeSSIF was prepared by first dissolving $16.5 \mathrm{~g}$ sodium taurocholate in $500 \mathrm{~mL}$ of blank FeSSIF, checking and noting the weight ("weight 1"). Subsequently, $59.1 \mathrm{~mL}$ of a methylene chloride solution containing $100 \mathrm{mg} / \mathrm{mL}$ lecithin (= $5.91 \mathrm{~g}$ lecithin,"weight 2 ") was added, resulting in an emulsion. The methylene chloride was then evaporated under the conditions described for FaSSIF until a clear, micellar solution with no perceptible odor of methylene chloride was obtained. After cooling to room temperature, the weight of the solution was checked again, and the water lost to evaporation was replaced with demineralized water to obtain a total weight corresponding to the sum of "weight 1" and "weight 2." Finally, the volume was brought to $2 \mathrm{~L}$ with blank FeSSIF. These methods of manufacturing FaSSIF and FeSSIF differed slightly from those proposed in the literature (11, 12) but, in the hands of the present investigators, resulted in micellar solutions with more reliable physicochemical parameters, particularly in terms of osmolality and ionic strength (13).

Simplified media containing different types and amount of surfactants were prepared using blank FaSSIF and FeSSIF (i.e., the relevant buffers without sodium taurocholate and lecithin) as the basis. After the surfactant(s) were added, the media were placed in the ultrasonic bath for $15 \mathrm{~min}$ and then stirred for another 15 min on a magnetic stirrer. Subsequently, various physicochemical parameters such as $\mathrm{pH}$, surface tension, critical micelle concentration, osmolality, and buffer capacity were measured and compared with those of the biorelevant media. Media with corresponding physicochemical properties (see Table 2 for the target values and the accepted tolerances) were then selected for solubility and dissolution experiments.

\section{Physical Chemical Evaluation of the Media}

The $\mathrm{pH}$ of all test media was measured with a $\mathrm{pH}$ meter. The buffer capacity was quantified by potentiometric titration with $0.1 \mathrm{~N}$ hydrochloric acid. The osmolality was measured by semi-micro osmometry (osmometer type ML, No. A0299, Knauer, Berlin, Germany), and the surface

Table 1. Composition and Physicochemical Properties of FaSSIF and FeSSIF.

\begin{tabular}{|c|c|c|c|}
\hline \multicolumn{2}{|c|}{ FeSSIF } & \multicolumn{2}{|c|}{ FaSSIF } \\
\hline Sodium taurocholate & $3 \mathrm{mM}$ & Sodium taurocholate & $15 \mathrm{mM}$ \\
\hline Lecithin & $0.75 \mathrm{mM}$ & Lecithin & $3 m M$ \\
\hline $\mathrm{NaH}_{2} \mathrm{PO}_{4}$ & $3.438 \mathrm{~g}$ & Acetic acid & $8.65 \mathrm{~g}$ \\
\hline $\mathrm{NaCl}$ & $6.186 \mathrm{~g}$ & $\mathrm{NaCl}$ & $11.874 \mathrm{~g}$ \\
\hline $\mathrm{NaOH}$ pellets & qs ad $\mathrm{pH} 6.5$ & $\mathrm{NaOH}$ pellets & $4.04 \mathrm{~g}$ \\
\hline Deionized water & qs ad 1 liter & Deionized water & qs ad 1 liter \\
\hline $\mathrm{pH}$ & 6.5 & $\mathrm{pH}$ & 5.0 \\
\hline Osmolality [mOsmol/kg] & $\sim 270$ & Osmolality [mOsmol/kg] & $\sim 670$ \\
\hline Buffer capacity $[\mathrm{mEq} / \mathrm{pH} / \mathrm{L}]$ & $\sim 12$ & Buffer capacity [mEq/pH/L] & $\sim 72$ \\
\hline Surface tension $[\mathrm{mN} / \mathrm{m}]$ & 54 & Surface tension $[\mathrm{mN} / \mathrm{m}]$ & 48 \\
\hline
\end{tabular}

Table 2. Physicochemical Parameters of the Test Media: Target Values and Tolerances.

\begin{tabular}{|c|c|c|c|c|}
\hline \multirow[b]{2}{*}{ Parameter } & \multicolumn{2}{|c|}{ Simulated FeSSIF } & \multicolumn{2}{|c|}{ Simulated FaSSIF } \\
\hline & target value & range of tolerance & target value & range of tolerance \\
\hline $\mathrm{pH}$ & 6.5 & \pm 0.1 & 5.0 & \pm 0.1 \\
\hline Osmolality [mOsmol/kg] & 270 & \pm 15 & 670 & \pm 15 \\
\hline Buffer capacity [mEq/pH/L] & 12 & \pm 2 & 72 & \pm 2 \\
\hline Surface tension [mN/m] & 54 & \pm 2 & 48 & \pm 2 \\
\hline
\end{tabular}


tension was determined with a bubble-pressure tensiometer (No. 100517A, Sita Messtechnik GmbH, Dresden, Germany).

\section{Selection of Model Drugs and Formulations}

In the first set of experiments, the BCS class II compound ketoconazole was used as a model drug. The solubility of the pure drug and the dissolution rate of a marketed ketoconazole formulation in the simplified dissolution media were compared with results in FaSSIF and FeSSIF (with and without addition of bile components). In the second part of the study, the applicability of the simplified media to other poorly soluble compounds was evaluated. Two additional BCS Class II drugs, namely the weak acid glyburide and the weak base tamoxifen, were used for this purpose. Another aspect of that part of the study was to prove the discriminatory power of the simplified test media. For the latter purpose, it would be optimal to use a set of "good" and "bad" batches of a well-characterized formulation and, in an ideal case, to also correlate dissolution results with the corresponding in vivo data. However, neither different batches of a formulation nor in vivo data were available at the time of the experiment. Thus, a compromise was to perform the release experiments with different qualities of a "self-made" capsule formulation containing mixtures of different ratios of the poorly soluble pure drug and partial tablets of the same drug. Prerequisite for such a procedure is a significant difference in dissolution performance between the pure drug and the tablet formulation. A test medium with sufficient discriminatory power should then enable the analyst to discriminate between the drug applied by means of the intact tablet and partial tablets mixed with different ratios of the pure drug.

\section{Dissolution Studies}

Drug release experiments were performed with USP Apparatus 2 (Paddle), DT 6 and DT 700, Erweka, Germany. Each vessel was filled with $500 \mathrm{~mL}$ of media, and an agitation speed of $75 \mathrm{rpm}$ was used for all dissolution studies. Experiments were run in triplicate. Samples $(5 \mathrm{~mL})$ were removed after $5,10,15,20,30,45,60,90$, and $120 \mathrm{~min}$ using a glass syringe, then filtered through a $0.45-\mu \mathrm{m}$ Teflon ${ }^{\circledR}$ filter and immediately diluted with methanol.

\section{HPLC Analysis}

All samples were analyzed by HPLC. The detailed HPLC conditions are specified in Table 3.

\section{RESULTS AND DISCUSSION}

As a first step, drug release from Nizoral ${ }^{\circledR}$ tablets was determined in the biorelevant media FaSSIF and FeSSIF and their corresponding blank buffers (see Figure 1).

In the second step, the aim was to generate media with physical chemical properties corresponding to those obtained in FaSSIF and FeSSIF. Measurement of the physicochemical parameters indicated that $\mathrm{pH}$, osmolality, and buffer capacity of blank FaSSIF and blank FeSSIF are not influenced by the presence of the surfactants tested. In contrast and as expected, the surface tension of the media prepared with various synthetic surfactants was markedly influenced by the type and concentrations of surfactants (see Figure 2). Concentrations above the critical micelle concentration (CMC) were used for all experiments. To adequately reflect the physicochemical parameters of FaSSIF and FeSSIF, only those surfactants resulting in surface tensions of $52-56 \mathrm{mN} / \mathrm{m}$ in blank FaSSIF or $46-50 \mathrm{mN} / \mathrm{m}$ in blank FeSSIF were selected for subsequent solubility and dissolution experiments.

The major intention of the present series of tests was to adequately reflect the physicochemical parameters of FaSSIF and FeSSIF. Some synthetic surfactants, several of which are prescribed in compendial dissolution test

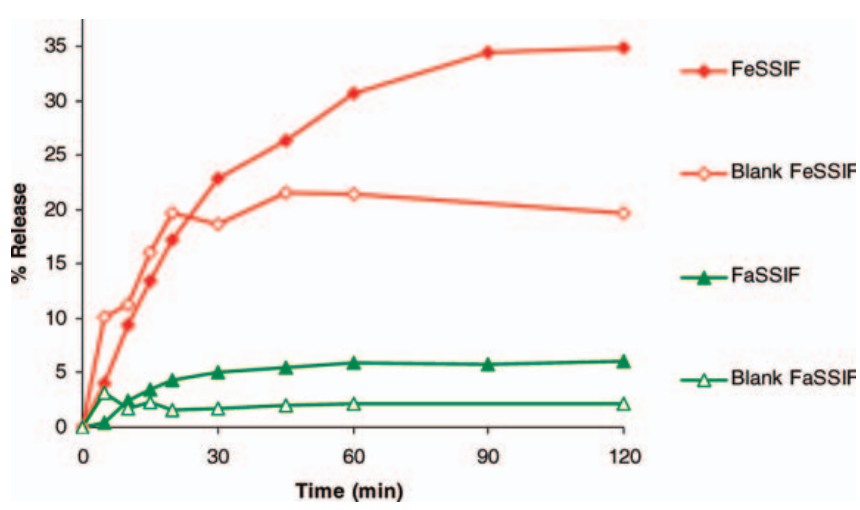

Figure 1.Drug release from Nizora ${ }^{\circledR}$ tablets in biorelevant media and their corresponding blank buffers.

\section{Table 3. HPLC Conditions.}

\begin{tabular}{|c|c|c|c|c|}
\hline Active substance & Column & Mobile Phase & Flow rate & Detection wavelength \\
\hline Ketoconazole & $\begin{array}{l}\text { Lichrocart }{ }^{\circledR} \text { RP-18, } 5-\mu \mathrm{m} 125 \times 4 \mathrm{~mm} \\
\text { (Merck, Darmstadt, Germany) }\end{array}$ & $\begin{array}{l}55 \% \text { acetonitrile } 45 \% \text { purified water } 0.02 \% \\
\text { triethylamine }\end{array}$ & $1.2 \mathrm{~mL} / \mathrm{min}$ & $254 \mathrm{~nm}$ \\
\hline Glyburide & $\begin{array}{l}\text { C-18 ET 125/4 Nucleosil } 120 \times 5 \text { mm } \\
\text { (Macherey-Nagel, Dueren, Germany) }\end{array}$ & $\begin{array}{l}65 \% \text { acetonitrile } 35 \% \text { purified water adjusted to } \\
\mathrm{pH} 3.0 \text { with phosphoric acid }\end{array}$ & $1.0 \mathrm{~mL} / \mathrm{min}$ & $230 \mathrm{~nm}$ \\
\hline Tamoxifen & $\begin{array}{l}\text { Lichrocart }{ }^{\circledR} \text { RP-18, } 5-\mu m 125 \times 4 \text { mm } \\
\text { (Merck, Darmstadt, Germany) }\end{array}$ & $\begin{array}{l}80 \% \text { acetonitrile } 19.9 \% \text { phosphoric acid } 15 \mathrm{mM} \\
0.01 \% \text { triethylamine }\end{array}$ & $1.0 \mathrm{~mL} / \mathrm{min}$ & $275 \mathrm{~nm}$ \\
\hline
\end{tabular}




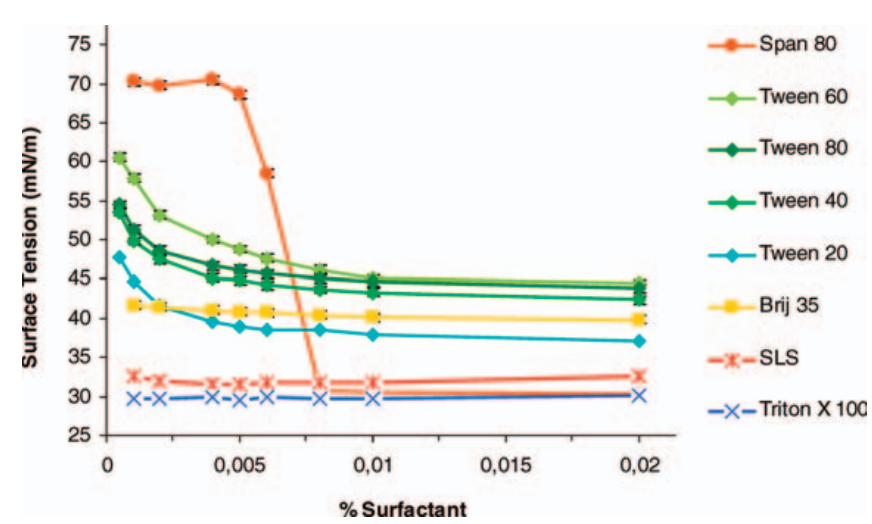

Figure 2. Surface tensions of various mixtures of surfactants with blank FaSSIF or blank FeSSIF (surface tension was independent of the buffer used).

methods, appeared to be unsuitable for the stated purpose since they lowered the surface tension too much. This was particularly the case for sodium dodecyl sulfate (SDS/SLS), which resulted in surface tensions of about $30 \mathrm{mN} / \mathrm{m}$ at concentrations above the CMC. This was, by far, too low for our purpose. On the other hand, the polysorbates Tween ${ }^{\circledR} 20,40,60$, and 80 appeared to be promising, since they resulted in surface tensions corresponding to that of FaSSIF or FeSSIF when used in concentrations of $0.05-0.5 \%$. Solubility of ketoconazole pure drug in several of the Tween-based media was similar to that in FaSSIF or FeSSIF, depending on concentration (data not shown). To examine whether these observations would be reflected in the dissolution behavior of a marketed formulation, dissolution experiments were performed in all media tested. Figure 3 illustrates the resulting drug release profiles.

Results from the dissolution experiments were in good agreement with those from the solubility studies. For ketoconazole, it was possible to simulate dissolution behavior under fasted-state small intestinal conditions using buffers containing simple surfactants like Tween ${ }^{\circledR} 60$ and 80 , while for fed state conditions, Tween ${ }^{\circledR} 20$ and 40 proved useful. As illustrated in Figure 3, blank FeSSIF containing a combination of $0.25 \%$ Tween ${ }^{\circledR} 80$ and $0.25 \%$ triethanolamine resulted in dissolution profiles almost superimposable to those in FeSSIF. Compared with media containing a single surfactant, this mixture seems to be more favorable because it is likely that mixed micelles are formed, which is analogous to the behavior of natural bile components (14). In this context, the use of triethanolamine might be somewhat surprising since the compound itself has no emulsifying properties. However, because it is both a tertiary amine and a tri-alcohol, it possesses hydrophilic and hydrophobic properties. The intention of its use was to simulate at least some functional domains of the lecithin molecule, thus facilitating the formation of mixed micelles and stabilizing them. However, this theory has to be proved in future

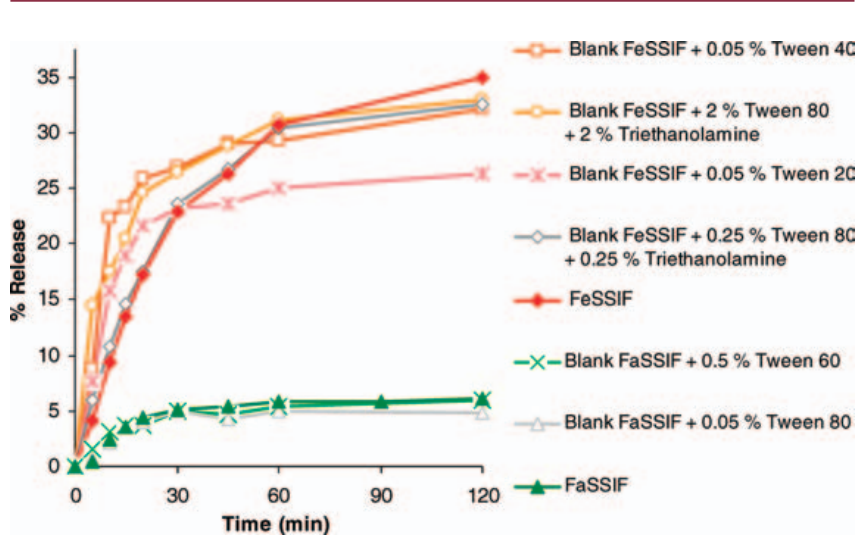

Figure 3. Drug release from Nizoral ${ }^{\circledR}$ tablets in simplified "biorelevant" dissolution media.

experiments. Although triethanolamine was the only compound evaluated for this purpose, it is quite likely that other compounds may better serve this function.

In a subsequent series of experiments, media consisting of blank FeSSIF with $0.25 \%$ Tween ${ }^{\circledR} 80$ and $0.25 \%$ triethanolamine, and blank FaSSIF with $0.05 \%$ Tween ${ }^{\circledR} 80$ were used to simulate FeSSIF and FaSSIF, respectively. No triethanolamine was used for the simplified FaSSIF, since in preliminary tests it had been shown that at this low concentration of Tween ${ }^{\circledR} 80$, addition of triethanolamine did not change the resulting profile. Despite the promising results obtained with Tween ${ }^{\circledR} 60$, no further experiments were performed using this surfactant. Due to the limited aqueous solubility of Tween ${ }^{\circledR} 60$, the preparation of such media was too time consuming, which was contradictory to the objective of the present study. The dissolution profiles shown in Figures 4-7 indicate that with the two media described above, it was possible to generate dissolution profiles similar to those obtained in FaSSIF and FeSSIF for both glyburide and tamoxifen citrate formulations. Furthermore, the discriminatory power of the two media was confirmed since in all cases, it was possible to distinguish between the different compositions of the formulations tested.

In the glyburide experiments, it was a bit difficult to distinguish between drug release from the complete tablet ( $3.5 \mathrm{mg}$ glyburide) and the mixture of $3 / 4$ tablet and $0.875 \mathrm{mg}$ pure drug. However, this was not remarkable since the saturation solubility in this setup is reached when only about $45 \%$ (simplified FaSSIF) or $30 \%$ (simplified FeSSIF) of the dose is released. Since the pure drug does not dissolve in the two media, the rate and extent of drug release of the mixtures is solely determined by drug release from the tablet portion. Thus, as long as more than half of a tablet is available, the profile is likely to reach its maximum level.

Tamoxifen citrate formulations represented better candidates for the objective of confirming the discriminatory power of the simplified test media. Whereas 


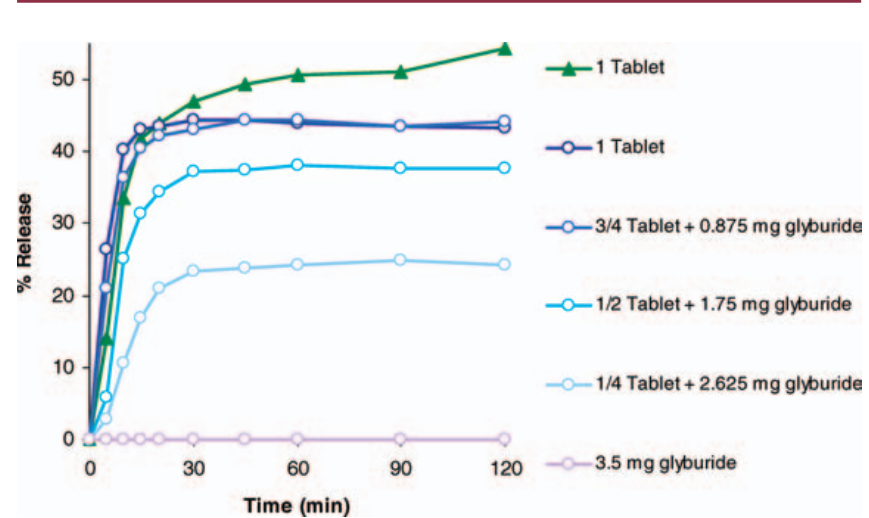

Figure 4. Drug release of Euglucon N, Euglucon N/glyburide mixtures, and glyburide in media simulating the fasted state: $\Delta$ FaSSIF, O: simplified FaSSIF, the test dose was $3.5 \mathrm{mg}$ glyburide in all cases.

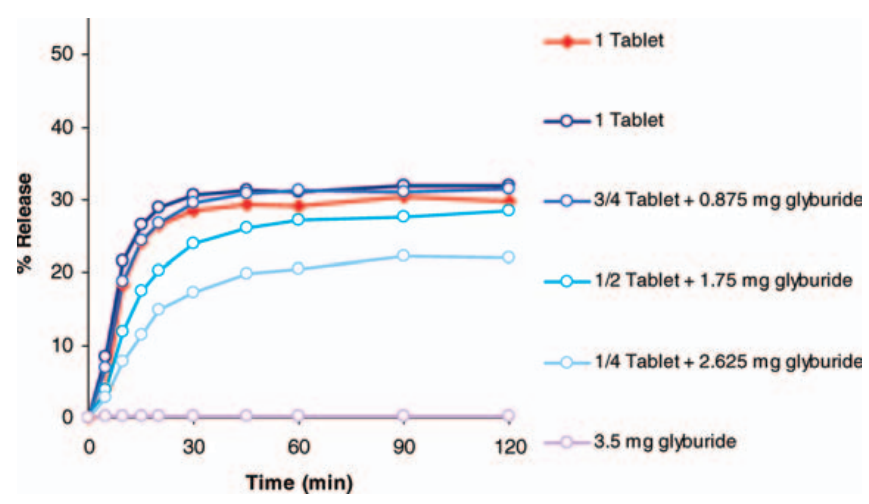

Figure 5. Drug release of Euglucon N, Euglucon N/glyburide mixtures and glyburide in media simulating the fed state: FeSSIF, O: simplified FeSSIF, the test dose was $3.5 \mathrm{mg}$ glyburide in all cases.

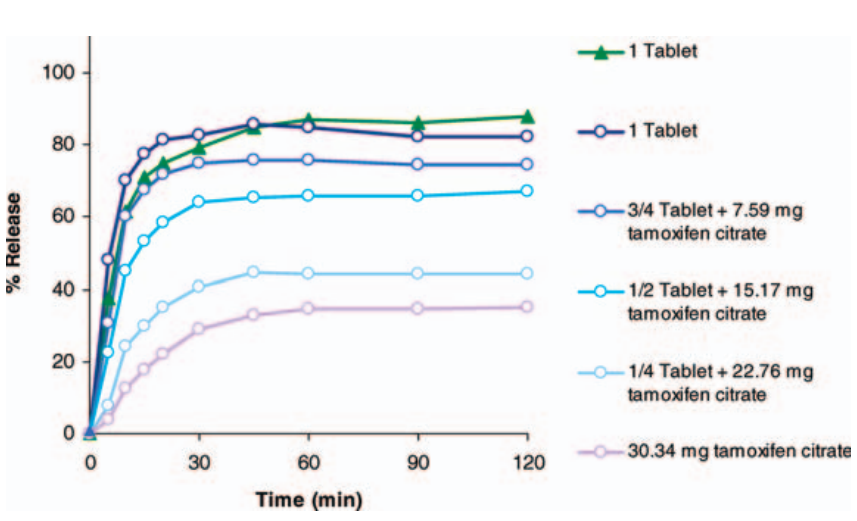

Figure 6. Drug release of Tamoxifen Hexal 20 mg, Tamoxifen Hexal/tamoxifen citrate mixtures, and tamoxifen citrate in media simulating the fasted state: - FaSSIF, O: simplified FaSSIF, the test dose was $30.34 \mathrm{mg}$ tamoxifen citrate $(\approx 20 \mathrm{mg}$ tamoxifen) in all cases.

almost $100 \%$ of drug was released from the tamoxifen citrate tablets, the pure drug showed a poor dissolution behavior in both biorelevant and simplified test media. For

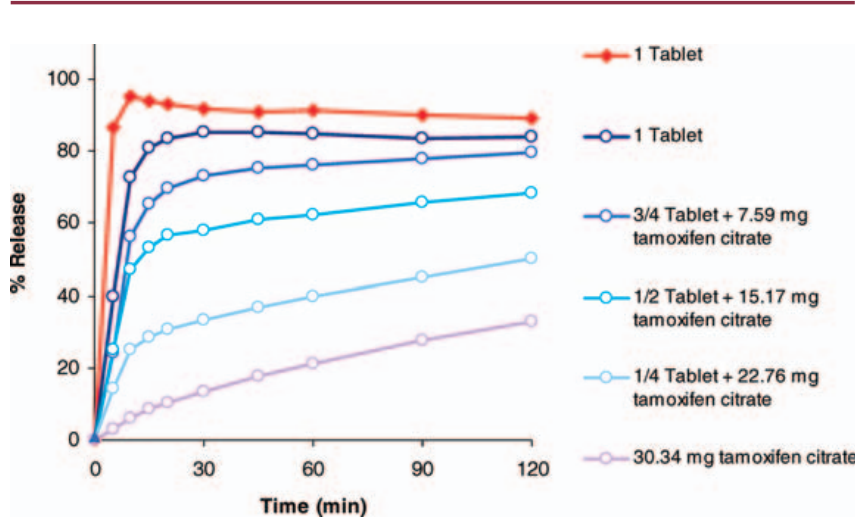

Figure 7. Drug release of Tamoxifen Hexal 20 mg, Tamoxifen Hexal/tamoxifen citrate mixtures, and tamoxifen citrate in media simulating the fed state: - FeSSIF, O: simplified FeSSIF, the test dose was 30.34 mg tamoxifen citrate $(\approx 20 \mathrm{mg}$ tamoxifen) in all cases

this reason, the difference in the dissolution profiles of the test formulations was more pronounced, and the rank order of the dissolution profiles clearly indicated the ratio of the ingredients in both simplified test media. Based on the observations made in the present series of tests, it is likely that these or similar media could be utilized to develop test methods for the early phases of formulation development and also have potential for "biorelevant" quality control (QC) tests. Additional advantages of their use include a much better storage stability, ease of preparation, and-probably the most significant criterion for everyday use-a far lower price. For example, one liter of FeSSIF can cost as much as US\$700, whereas the price of one liter of the media containing synthetic surfactant(s) is only around US\$1.

\section{SUMMARY}

Selected media containing synthetic surfactants with physicochemical properties similar to those of FaSSIF and FeSSIF were identified. These can be used to replace biorelevant test media for screening formulations if it can be shown that solubility and dissolution of the drug in these simplified media are similar to the profiles in biorelevant media prepared with bile components. Validation of the correspondence of results in media containing synthetic surfactants with those containing bile components is necessary on a case-by-case basis. In cases where results are similar, the simplified media represent a very promising approach to designing both formulation screening and quality control tests that are relevant to in vivo conditions, while retaining the cost and time efficiencies associated with use of synthetic surfactants. In particular, when taking into account several additional physiological parameters (i.e., pH, buffer capacity, and osmolality) in the fasted and fed small intestine, the results generated with these media should be more reliable in predicting the in vivo release behavior of a formulation than those generated with commonly used surfactant systems. 


\section{REFERENCES}

1. Galia, E.; Nicolaides, E.; Hörter, D.; Löbenberg, R.; Reppas, C.; Dressman, J. B. Evaluation of various dissolution media for predicting in vivo performance of class I and II drugs. Pharm. Res. 1998, 15 (5), 698-705.

2. Nicolaides, E.; Galia, E.; Efthymiopoulos, C.; Dressman, J. B.; Reppas, C. Forecasting the in vivo performance of four low solubility drugs from their in vitro dissolution data. Pharm. Res. 1999, 16 (12), 1876-1882.

3. Dressman, J. B.; Reppas, C. In vitro-in vivo correlations for lipophilic, poorly water-soluble drugs. Eur. J. Pharm. Sci. 2000, 11 (Suppl 2), 73-80.

4. Kostewicz, E. S.; Brauns, U.; Becker, R.; Dressman, J.B. Forecasting the oral absorption behavior of poorly soluble weak bases using solubility and dissolution studies in biorelevant media. Pharm. Res. 2002, 19 (3), 345-349.

5. Nicolaides, E.; Hempenstall, J. M.; Reppas, C. Biorelevant Dissolution Tests with Flow-Through Apparatus. Dissolution Technol. 2000, 7 (1), 8-11.

6. Nicolaides, E.; Symillides, M.; Dressman, J. B.; Reppas, C. Biorelevant dissolution testing to predict the plasma profile of lipophilic drugs after oral administration. Pharm. Res. 2001, 18 (3), 380-388.

7. Wei, H.; Lobenberg, R. Biorelevant dissolution media as a predictive tool for glyburide a class II drug. Eur. J. Pharm. Sci. 2006, 29 (1), 45-52.

8. Buchanan, C. M., Buchanan, N. L.; Edgar, K. J.; Little, J. L.; Malcolm, M. O.; Ruble, K. M.; Wacher, V. J.;Wempe, M. F. Pharmacokinetics of tamoxifen after intravenous and oral dosing of tamoxifen-hydroxybutenyl-betacyclodextrin formulations. J. Pharm. Sci. 2007, 96 (3), 644-660.

9. Löbenberg, R.; Shah, V.; Krämer, J.; Amidon, G. L.; Dressman, J. B. Dissolution testing as a prognostic tool for oral drug absorption: dissolution behavior of glibenclamide. Pharm. Res. 2000, 17 (4), 439-444.

10. Charman, W. N.; Porter, C. J.; Mithani, S.; Dressman, J. B. Physiochemical and physiological mechanisms for the effects of food on drug absorption: the role of lipids and pH.J. Pharm. Sci. 1997, 86 (3), 269-282.

11. Galia, E. Physiologically based dissolution testsExperiences with poorly soluble drugs. Ph.D. Dissertation, Johann Wolfgang Goethe University, Frankfurt, 1999.
12. Marques, M. Dissolution Media Simulating Fasted and Fed States. Dissolution Technol. 2004, 11 (2), 16.

13. Klein, S. Biorelevant Dissolution Test Methods for Modified Release Dosage Forms. Ph. D. Dissertation, Johann Wolfgang Goethe University, Frankfurt, 2005.

14. Carey, M. C.; Small, D. M. Micelle formation by bile salts. Physical-chemical and thermodynamic considerations. Arch. Intern. Med. 1972, 130 (4), 506-527. 